\title{
In-situ conversion of rGO from graphene oxide based on solar mediated enhanced characterization properties
}

\author{
Subramani shanmugam, Sivanandan nanjan \\ Department of Electronics, PSG College of Arts and Science, Coimbatore- 641014, India \\ sai.subbu29@gmail.com, sivanandannanjan@gmail.com
}

DOI 10.17586/2220-8054-2019-10-5-579-584

\begin{abstract}
A globally acknowledged green synthesis of reduced graphene oxide (rGO) from graphene oxide (GO) is presented in this paper. The graphene oxide powder was synthesized from Graphite powder by a modification of Hummer's method. The GO is exposed to focused sunlight to obtain reduced graphene oxide (rGO). The reduction of GO under solar light is an eco-friendly method to conventional method of rGO preparation. The mechanism of the reduction of GO by sunlight imperative to exfoliation was seen to be well defined. The rGO powder was characterized by Xray Diffraction (XRD), Field-Emission Scanning Electron Microscopy (FESEM), Raman spectroscopy, Fourier-Transform Infrared Spectroscopy (FTIR) and High-Resolution Transmission Electron Microscopy (HRTEM). This eco-friendly method of synthesizing of rGO paves way for an alternative method of rGO nanosheets preparation and it can be effectively used for fabrication of various electronic devices.
\end{abstract}

Keywords: reduced graphene oxide, crystal structure, reduction, exfoliation, raman.

Received: 4 July 2019

Revised: 5 September 2019

\section{Introduction}

Graphene Oxide (GO) is being single-layered carbon atom in a hexagonal lattice has a combination of both aromatic ( $\mathrm{sp}^{2}$ hybridized $\mathrm{C}-\mathrm{C}$ atoms) and aliphatic $\left(\mathrm{sp}^{3}\right.$ hybridized $\left.-\mathrm{OH}, \mathrm{C}-\mathrm{O}-\mathrm{H}, \mathrm{C}=\mathrm{O},-\mathrm{COOH}\right)$ groups [1-3]. Thus, it exhibits a wide range of striking properties like optical transmittance, electrical conduction, energy storage, medical diagnosis and treatment, electronic applications, etc. The properties of GO and its reduced form plays a vital role in the multi-disciplinary functionality of these materials. They possess high surface area to volume ratio, high tensile strength, good thermal conduction, tremendous electrical conduction and superb optical properties [4]. The single layer GO and rGO can be synthesized by several methods, such as mechanical exfoliation, electrochemical reactions, thermal treatment and [5], photocatalytic method of preparing of GO and rGO. The solar energy assisted route of synthesis has an advantage of enhancement in its performance, especially when used for energy storage devices and super capacitors. Currently, a metallic oxide in combination with rGO and carbon nanotubes (CNTs), enhances the network performance of the devices. Oxides of tin, zinc, and cobalt are coated on graphene to obtain the desired quality of electrical devices [6-9]. Here, the purest form of solar energy was used to reduce the GO into rGO and this product was thoroughly characterized.

\section{Experimental}

\subsection{Materials and methods}

GO was synthesized from graphite powder using a modification of Hummer's method [10]. The reduced GO was prepared by using a convex lens of $100 \mathrm{~mm}$ diameter to focus solar radiation [11]. Briefly, $1 \mathrm{~g}$ of GO was taken in a glass Petri dish and kept under focused solar radiation at 12 Noon. GO powder was exposed to the focused solar radiation for approximately $10-15$ minutes. GO was exfoliated into rGO sheets. The power of focused sunlight ranged from $1.8-2.5 \mathrm{~W}$ and the temperature was about $\sim 300^{\circ} \mathrm{C}$ [12]. GO was exfoliated into rGO sheets under this high temperature and power [13].

\section{Result and discussion}

\subsection{XRD Analysis}

The XRD pattern of synthesized GO/rGO shows diffraction with $\mathrm{Cu} \mathrm{K} \alpha$ radiation $(\lambda=1.54178 \AA)$ and depicted in Fig. 1 [14]. The GO/rGO exhibits a well-crystalline orthorhombic crystal structure (JCPDS card No. 89-8491).

The GO/rGO featured sharp diffraction peaks indicates that GO at 11.83, 42.78 corresponds to (001) and (100) peaks. The diffraction peaks of rGO at 10.30, $23.31 \& 43.76$ correspond to (001), (002) \& (100) [15]. The diffraction 


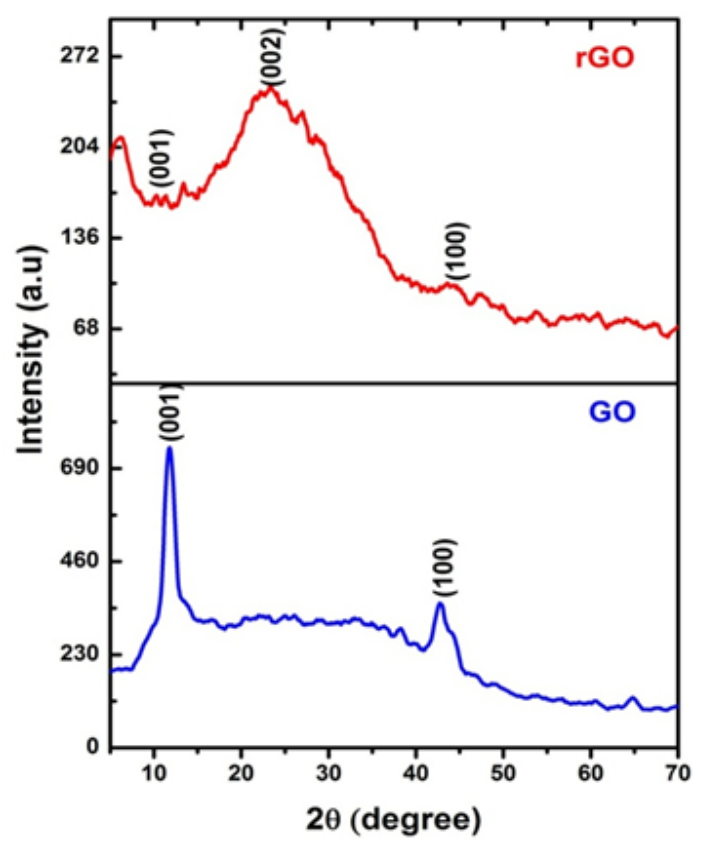

FIG. 1. X-ray diffraction pattern of GO and rGO nanosheets

plane of the hexagonal crystal structure has lattice constants of value, $a=2.47$ and $c=6.79 \AA$. The crystalline size $(D)$ of the $\mathrm{GO} / \mathrm{rGO}$ was calculated using Scherrer's formula:

$$
D=\frac{0.89 \lambda}{\beta \cos \theta},
$$

where $\lambda$ is the wavelength of the $\mathrm{X}$-ray radiation, $2 \theta$ is the diffraction angle and $\beta$ is the full width at half maximum (FWHM). Measured broadening of the GO/rGO with (001), (100) and 001),(002), (100) X-ray peaks offers average crystalline size about at 72.97, 14.03, and 19.59, 14.31, $21.23 \mathrm{~nm}$ for the GO/rGO, respectively [16].

\subsection{FESEM/EDS}

The morphology of GO/rGO nanosheets was observed using FESEM and shown in Fig. 2(a-b). The morphological feature clearly shows the formation of $\mathrm{GO} / \mathrm{rGO}$ in 2D sheet-like pattern morphology in the prepared graphene materials similar to commercial materials. 2D wrinkled structure morphology of both GO and rGO sheets were observed to be fine micrometer in size. The rGO has a few broad and highly wrinkled layers with a regular shape and slanting size estimated in the range size of approximately $300-500 \mu \mathrm{m}$ exclusively $[17,18]$.

The Energy Dispersive X-Ray Spectroscopy (EDS) analysis of the GO and rGO reveals that the atomic percentage of oxygen was considerably reduced in $\mathrm{rGO}$ than $\mathrm{GO}$ as expected. This is also confirmed by the increased ratio of $\mathrm{C} / \mathrm{O}$ from 1.6684 to 2.0075 .

\subsection{HRTEM}

Transmission electron microscopic (TEM) study was carried out to obtain the wide-ranging morphological information about the GO/rGO nanosheets and the respective HRTEM images were also showed in Fig. 3(a-d). The TEM image of GO/rGO confirms the nanosheets like morphology with their wrinkle shape with the face end (Fig. 3(a, b)). However, the TEM images of GO/rGO exhibits sheet-like shape with an average size of about $0.2 \mu \mathrm{m}$. In order to obtain that lattice fringes form in the plane (100) indicated, the d-spacing values in three different places are shown in Fig. 3(b). Inset images shows good conformity with TEM images [19].

A clear morphology of rGO was observed, as evidence that is shown in Fig. 3(c). The sheets of rGO obtained by the chemical reduction process possess a few layer forms, such as crumpled sheet-like morphology and the structure were further confirmed by TEM, as shown in Fig. 3(c, d). The uniform and wrinkled sheet-like morphology, as well as the micropores on sheet surface of rGO samples (Fig. 3(c)) were clearly observed. Also, it displays highly interconnected ultrathin silk-like morphology and wrinkled shape [20]. Moreover, the wrinkle of the layers folded over each other was clearly visible, suggesting that these wrinkles were occurred by the crumpling of graphene-like sheets. The results also show that the highly crumbled morphology can effectively prevent the aggregation of the sheets as 

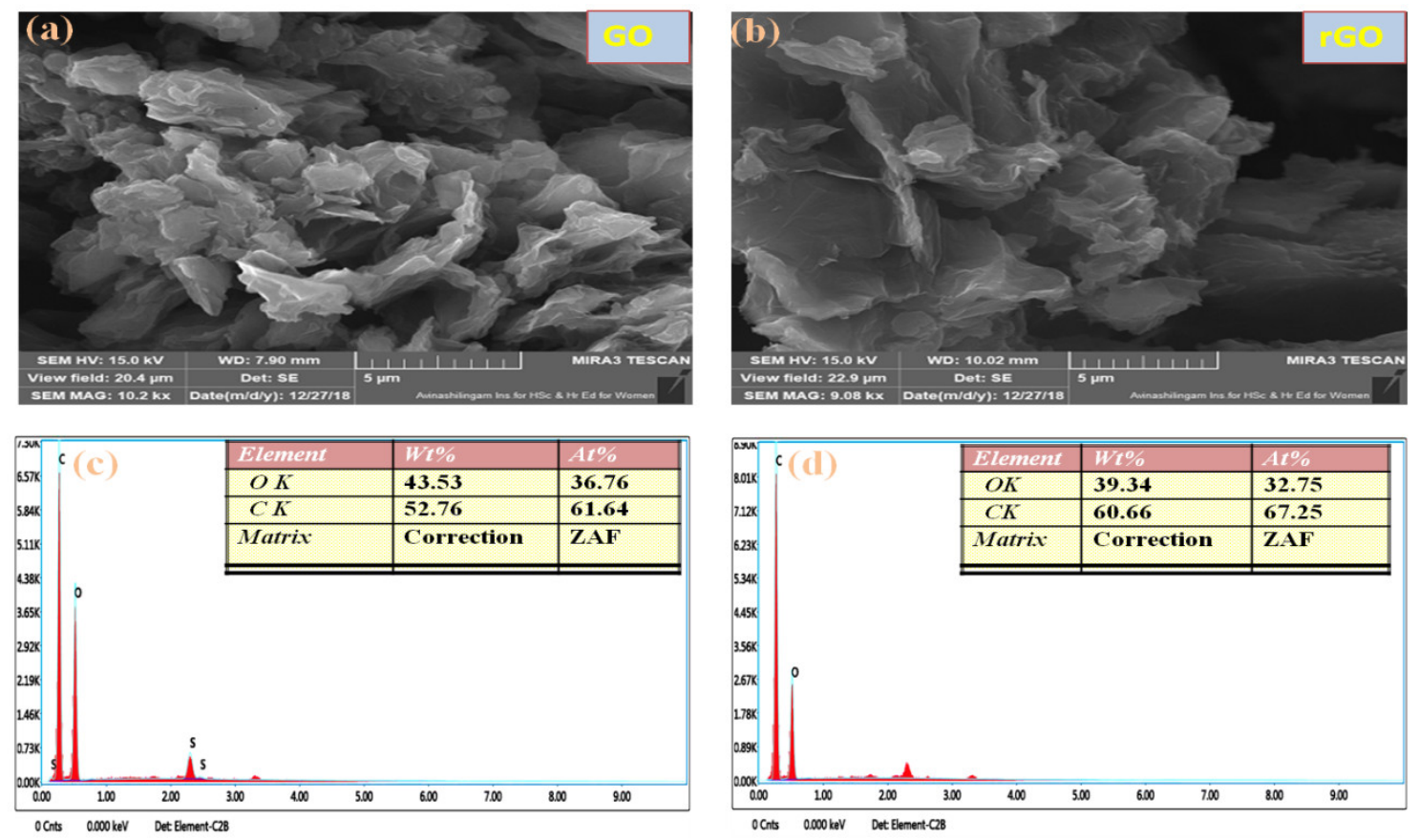

FIG. 2. FESEM surface morphology of GO and rGO nanosheets
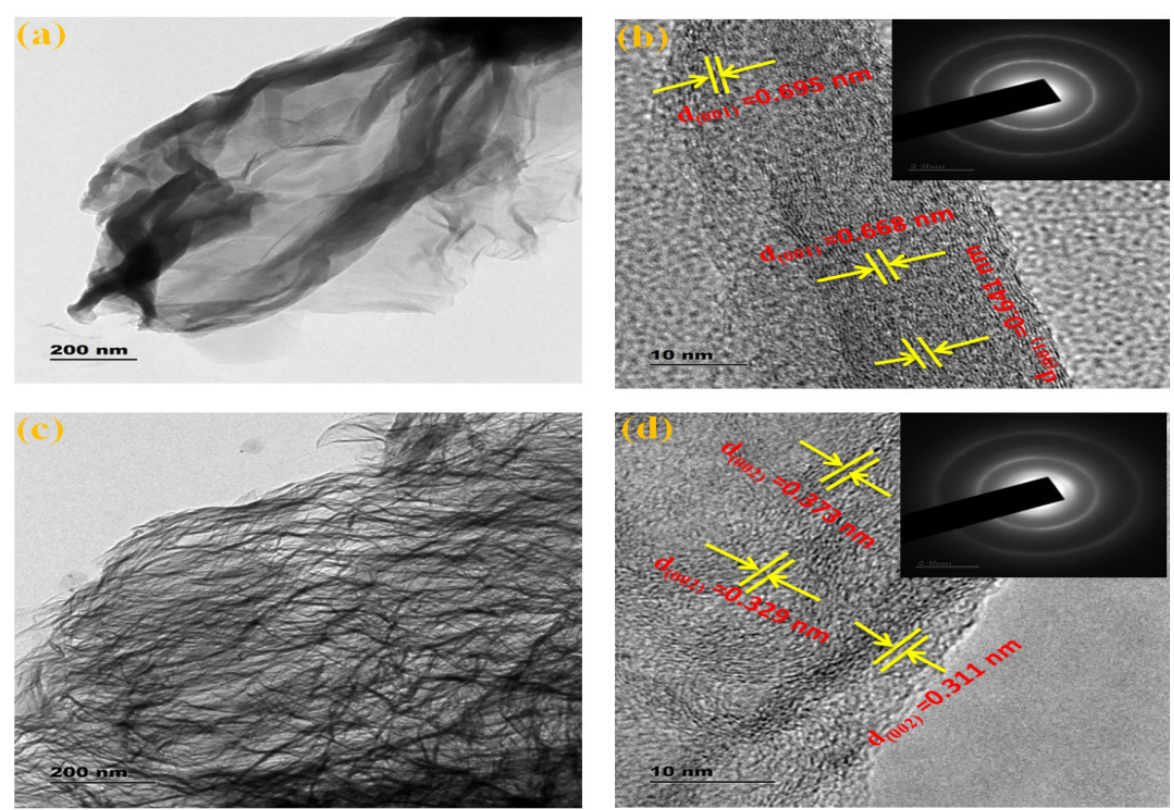

FIG. 3. HRTEM micrographs of GO and rGO nanosheets 
shown in Fig. 3(d). The lattice fringes form was correlated with the reports in the plane about (002) as indicated in the d-spacing value. Inset image Fig. 3(d) clearly shows a well standard SAED pattern in two ring shape with a circle in the poly crystalline nature of the rGO [21]. The HRTEM result is in agreement with the XRD result in Fig. 2(d). These results conclude the morphology of the prepared $\mathrm{GO} / \mathrm{rGO}$ [22].

\subsection{Raman spectroscopy}

The structure of GO/rGO was studied using Raman spectroscopy. The GO/rGO nanosheets exhibited a series of bands at 143, 493, 625, 687, 847, 1172, 1369, 1617, 1840, 1980, 2234 and $2336 \mathrm{~cm}^{-1}$. All these bands were attributed to the fundamental vibration modes existing in graphene oxides. The reduction of GO and rGO sheets were further confirmed through Raman spectra as shown in Fig. 4. The Raman spectra of GO displays two characteristic Raman bands centered at 1369 and $1617 \mathrm{~cm}^{-1}$ were assigned to the well-known D and $\mathrm{G}$ bands, respectively [23].

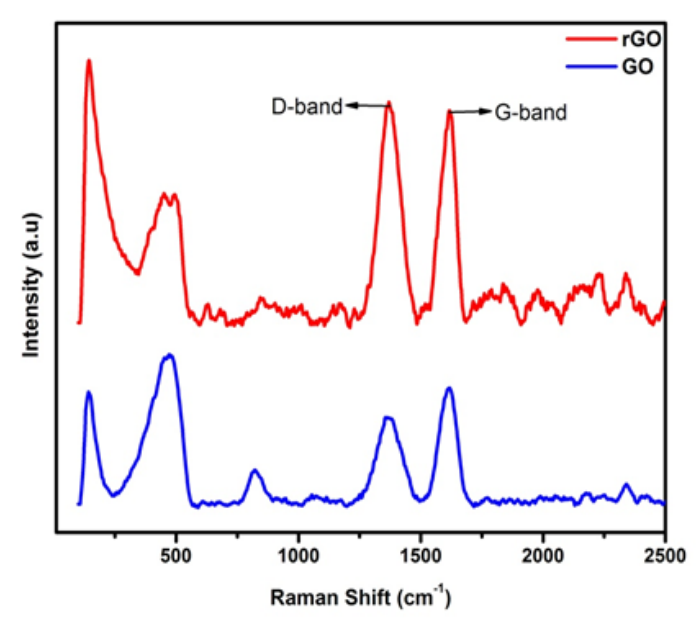

FIG. 4. Raman spectra of GO and rGO nanosheets

The $\mathrm{D}$ band $\left(\sim 1369 \mathrm{~cm}^{-1}\right)$ corresponds to disorder in the $\mathrm{sp}^{2}$ carbon network, and the $\mathrm{G}$ band $\left(\sim 1617 \mathrm{~cm}^{-1}\right)$ is associated with the tangential vibrations of the $\mathrm{sp}^{2}$ carbon atoms in the hexagonal planes. In general, the $\mathrm{D}$ band is known as an imperfect band that typically arises from the first order diffusion of $\mathrm{sp}^{3}$ hybridized carbon atoms, while the $\mathrm{G}$ band is mainly reflects from the stretching vibration of $\mathrm{sp}^{2}$ hybridized $\mathrm{C}=\mathrm{C}$ bonds. Results show that there is a shift occurring in Raman peaks positions for pure graphene oxide, which confirms the bond formation between graphene layers of rGO. The ID/IG ratio of the D-band to the G-band is related to the disorderliness of the $\mathrm{sp}^{2}$ domain, owing to the removal of the oxygen functional groups (Fig. 4) [24]. The ID/IG ratios for GO and rGO were found to be 0.750 and 1.030 respectively (Fig. 4).

\subsection{FTIR spectrum}

The major difference between the GO and rGO depicts the presence of specific functional groups like hydroxyl $(-\mathrm{OH})$, carboxyl $(\mathrm{C}=\mathrm{O})$, epoxy $(\mathrm{C}-\mathrm{O}-\mathrm{C})$ molecules attached to the main graphene layer [25]. These groups show the molecular vibrations of a specific characteristic frequency, when exposed to infrared radiation, as shown by the FTIR study in Fig 5.

The GO shows a sharp peak at $1040 \mathrm{~cm}^{-1}$ (representing C-O-C), $1713 \& 1607 \mathrm{~cm}^{-1}$ (corresponding to C=O) and a board peak around $3000 \mathrm{~cm}^{-1}$ (attributed to $-\mathrm{OH}$ groups) apart from oxygen base plane [26]. These have become less broadened with rGO samples due to the reduction in the concentration of vibrational groups.

\subsection{PL Spectrum}

The PL spectrum of GO nanosheets are depicted in Fig 6(a). The photoluminescence characterized by a broad peak between $600-900 \mathrm{~nm}$ was observed and the emission sharp peak was detected in GO at $750 \mathrm{~nm}$. Fig. 6(b) shows PL spectra of rGO.

Three emission peaks was observed at 350, 435 and $524 \mathrm{~nm}$, respectively, that are observed in rGO and it is attributed to the recombination of electron hole pairs in local state of $\mathrm{sp}^{2}$ carbon cluster embedded in $\mathrm{sp}^{3}$ matrix [27], in which are clearly known as $\mathrm{GO}$ is reduced to become rGO. 


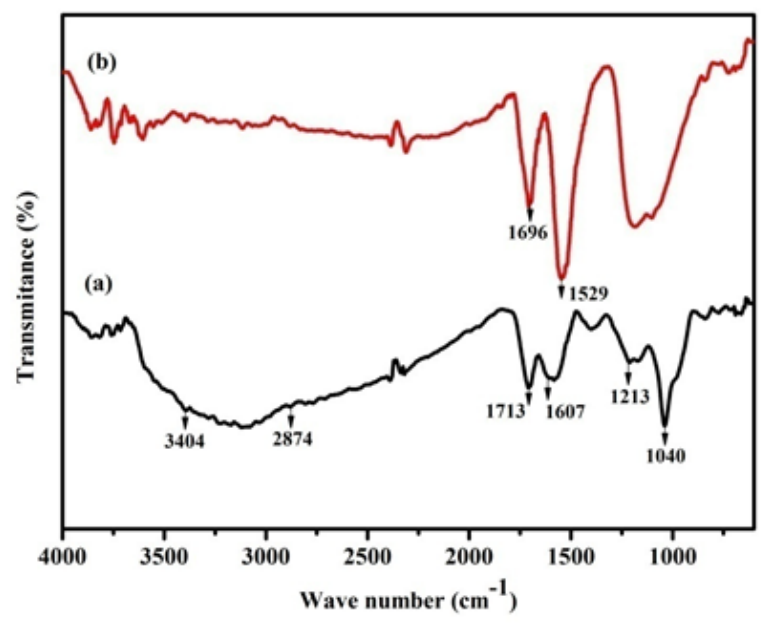

FIG. 5. FTIR spectra of (a) GO and (b) rGO nanosheets
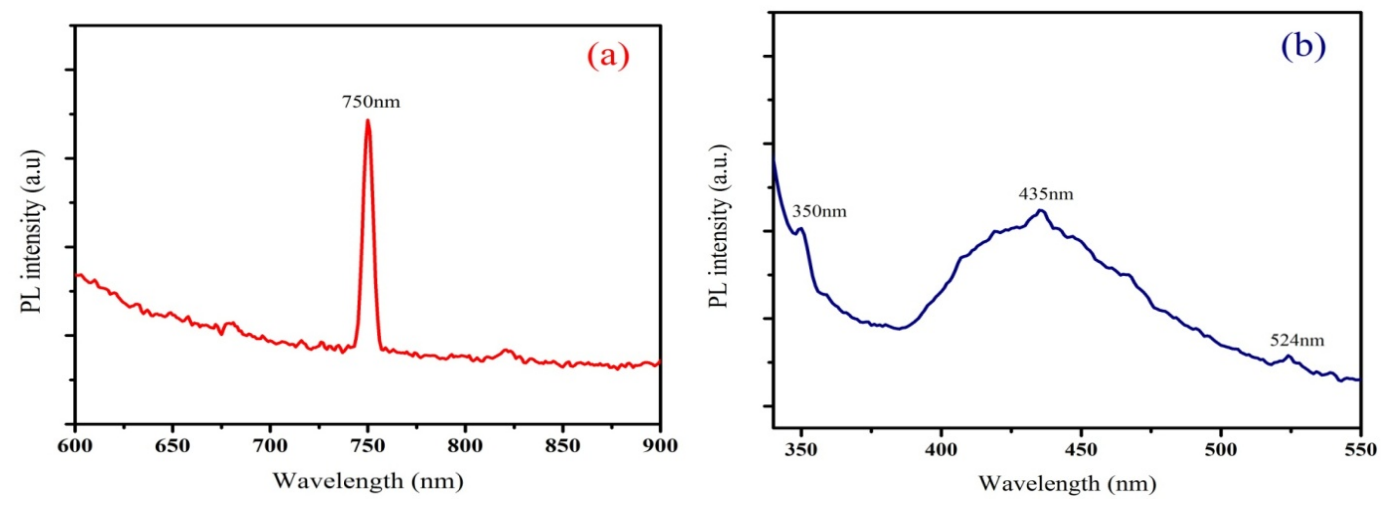

FIG. 6. PL spectrum of (a) GO and (b) rGO nanosheets

\section{Conclusions}

To summarize, we have successfully synthesized and characterized GO/rGO nanosheets. The crystalline structures of GO/rGO have been determined by XRD measurements. The morphological analysis shown by FESEM exhibits conspicuous wrinkled flakes. HRTEM images studies shows SAED patterns and nanosheets like structure in the plane with (002) orientation. The weight percentage of oxygen was reduced in $\mathrm{GOO}$, as compared to GO which confirms the reduction and the formation of reduced graphene oxide. Raman spectra inferred that D/G value of GO $0.750(<1)$ and $\mathrm{rGO} 1.030(>1)$ respectively, which confirms the formation of GO and rGO. Thus, the shallow peaks of rGO observed in FTIR reveals the partial removal of oxygen-linked functional groups from the hexagonal carbon-carbon plane of GO. The PL spectra of GO/rGO peak at 750 and $435 \mathrm{~nm}$ shows red and blue shift. Design of hetero-junction photodiode fabrication could be a thought for future amelioration and future scope of this research work. Due to its simplicity, this eco-friendly method of synthesizing rGO paves the way for making enormous rGO supported devices with good characteristics.

\section{References}

[1] Anand K., Singh O., et al. Hydrogen sensor based on graphene/ZnO nanocomposite. Sens. Actuators B, 2014,195 , P. $409-415$.

[2] Ning G., Fan Z., et al. Gram-scale synthesis of nanomesh graphene with high surface area and its application in supercapacitor electrodes. Chem. Commun., 2011, 47, P. 5976-5978.

[3] Lee V., Whittaker L., Jaye C., Baroudi K.M. Large-area chemically modified graphene films: Elecrophoretic deposition and characterization by soft X-ray absorption spectroscopy. Chem. Mater, 2009, 21 (16), P. 3905-3916.

[4] Veldevi T., Thileep Kumar K., et al. Synthesis of Hierarchical graphene- $\mathrm{MnO}_{2}$ nanowire composites with enhanced specific capacitance. Asian J. Chem., 2019, 31 (8), P. 1709-1718.

[5] Mahanandia P., Simon F., Heinrich G., Nanda K.K. An electrochemical method for the synthesis of few layer graphene sheets for high temperature applications. Chem. Commun., 2014, 50 (35), P. 4613-4615. 
[6] Zhou Y., Ma L., et al. Mono disperse $\mathrm{MnO}_{2} @ \mathrm{NiCo}_{2} \mathrm{O}_{4}$ core/shell nanospheres with highly opened structures as electrode materials for good-performance super capacitors. Appl. Surf. Sci., 2018, 444, P. 1-9

[7] Sahoo S., Shim J.J. Nanostructured 3D zinc cobaltite/nitrogen-doped reduced graphene oxide composite electrode for supercapacitor applications. J. Ind. Eng. Chem., 2017, 54, P. 205-217.

[8] Zhou X., Shen X., et al. Hollow Fluffy $\mathrm{Co}_{3} \mathrm{O}_{4}$ Cages as Efficient Electro active Materials for Super capacitors and Oxygen Evolution Reaction. ACS Appl. Mater. Interface, 2015, 7, P. 20322-20331.

[9] Pawar S.A., Patil D.S., Shin J.C. Hexagonal sheets of $\mathrm{Co}_{3} \mathrm{O}_{4}$ and $\mathrm{Co}_{3} \mathrm{O}_{4}-\mathrm{Ag}$ for high-performance electrochemical super capacitors. J. Ind. Eng. Chem., 2017, 54, P. 162-173.

[10] Hummers W.S., Offeman R.E. Preparation of Graphitic oxide. J. Am. Chem. Soc., 1958, 80 (6), P. $1339-1339$.

[11] Aravind S.S.J., Eswaraiah V., Ramaprabhu S. Facile and simultaneous production of metal/metal oxide dispersed graphene nano composites by solar exfoliation. J. Mater. Chem., 2011, 21, P. 17094-17097.

[12] Mohandoss M., Gupta S.S., et al. Solar mediated reduction of graphene oxide. RSC Adv., 2017, 7, P. $957-963$.

[13] Thangavel P., Kannan R., et al. Development of reduced graphene oxide (rGO)-isabgol nano composite dressings for enhanced vascularization and accelerated wound healing in normal and diabetic rats. J. Coll. Inter. Sci., 2018, 517, P. 251-264.

[14] Murugandi G., Saravanan M., et al. Barium borate nanorod decorated reduced graphene oxide for optical power limiting applications. Opt. Mat., 2018, 75 (2018), P. 612-618.

[15] Chakraborty A., Agresti A., et al. Wet-Chemical Synthesis of ZnO Nanowires on Low Temperature Photo-Activated ZnO-rGO Composite Thin Film with Enhanced Photoconduction. J. Materresbull., 2018, 47, P. 5863-5869.

[16] Meng H., Yang W., et al. $\mathrm{Cu}_{2} \mathrm{O}$ nanorods modified by reduced graphene oxide for $\mathrm{NH}_{3}$ sensing at room temperature. J. Mater. Chem. A, 2015, 3, P. 1174-1181.

[17] Babeela C., Sabari Girisun T.C. Advances on the fabrication process of $\mathrm{Er}^{3+} / \mathrm{Yb}^{3+}: \mathrm{GeO}_{2}-\mathrm{PbO}$ pedestal waveguides for integrated photonics. Opt. Mater., 2015, 49, P. 190-200.

[18] SabariGirisun T.C., Madura Somayaji R., Priyadarshini N., Venugopal Rao S. Femto third order optical nonlinearity and optical limiting studies of $(\gamma$ and $\beta$ ) Barium borate nanostructures. Mater. Res. Bull., 2017, 87, P. 102-108.

[19] Gao G., Wu H.B., et al. Growth of ultrathin $\mathrm{ZnCo}_{2} \mathrm{O}_{4}$ nanosheets on reduced graphene oxide with enhanced lithium storage properties. Adv. Sci., 2015, 2, 1400014.

[20] Roy M., Kusurkar T.S., et al. Graphene oxide from silk cocoon: a novel magnetic fluorophore for multi-photon imaging. 3Biotech., 2014, 4, P. 67-75.

[21] Sun Z., Yan Z., et al. Growth of graphene from solid carbon sources. Nature, 2010, 468, P. 549-552.

[22] Bo Z., Shuai X., et al. Green preparation of reduced graphene oxide for sensing and energy storage applications. Sci.ReP., 2014,4 , P. 4684.

[23] Liu H., Xu B., et al. Polyaniline nanofiber/large mesoporous carbon composite as electrode materials for super capacitors. Appl. Surf. Sc., 2015, 332, P. 40-46.

[24] Fan Z.J., Kai W., et al. Facile synthesis of graphene nanosheers via Fe reduction of exfoliated graphite oxide. ACS nano, 2011, 5 (1), P. 191198.

[25] Sengupta I., Chakraborty S., et al. Thermal reduction of graphene oxide: How temperature influences purity. J. Mater. Res., 2018, 33 (23), P. 4113-4122.

[26] Etacheri V., Yourey J.E., Bartlett B.M. Chemically bonded $\mathrm{TiO}_{2}$-bronzenanosheet/reduced graphene oxide hybrid for high-power lithium ion batteries. ACS nano, 2014, 8 (2), P. 1491-1499.

[27] Zhu Y.W., Murali S., et al. Graphene and graphene oxide: synthesis, properties and applications. Adv. Mater., 2010,22 , P. $3906-3924$. 